\title{
Finite simple groups with no elements of order six
}

\section{L.M. Gordon}

\begin{abstract}
The aim of this paper is to completely classify the finite simple groups with no elements of order 6 . The proof is by induction and involves an analysis of the structure of the 2-local subgroups of a counterexample of minimum order. A recent result of Glauberman plays an essential role.
\end{abstract}

The object of this paper is to completely classify the groups of the title. The result has recently been obtained independently by Fletcher, Stel Imacher, Stewart [4], but the author feels that the present proof has independent interest.

THEOREM. Let $G$ be a finite simple group which has no elements of order six. Then $G$ is isomorphic to one of the following groups:

$$
\begin{aligned}
& \text { (i) } \operatorname{PSL}(2, q), q \neq 1,11 \bmod 12 ; \\
& \text { (ii) } \operatorname{PSL}\left(3,2^{n}\right), n \geq 2, n \neq 0 \bmod 6 ; \\
& \text { (iii) } \operatorname{PSU}\left(3,2^{n}\right), n \geq 2, n \neq 3,5 \bmod 6 \text {; } \\
& \text { (iv) } S z\left(2^{2 n+1}\right), n \geq 1 .
\end{aligned}
$$

The centralizer of any 3-element in $G$ has odd order whence the main theorem could be rephrased to give an "odd characterization" of the simple groups listed.

Notation is essentially standard (see [5], [6], [9]). For a set $V$,

Received 19 April 1977. The author would like to express his thanks to Dr D.E. Taylor for many valuable suggestions and his kind encouragement. 
$O(V)$ will denote the cardinality of $V$. In particular, $O(H)$ is the order of a group $H$ and if $x \in H, o(x)=o(\langle x))$. If $H$ is a finite group then sol $(H)$ is the maximal soluble normal subgroup of $H, \operatorname{soc}(H)$ is the product of the minimal normal subgroups of $H$, and $L(H)$ is the maximal normal semisimple subgroup of $H$.

For the remainder of the paper, $G$ will denote a counterexample to the theorem of minimum order. We commence with an important observation. The hypothesis of the theorem is inductive to subgroups and sections and thus the only simple groups involved in $G$ are those listed in the theorem. Moreover, if $t$ is an involution of $G$ then $C_{G}(t)$ is soluble or involves only Suzuki groups and so $G$ is balanced ([16], p. 285). An inspection of the list of Gorenstein and Harada [11, Corollary A] shows that $\mathrm{SCN}_{3}(2)$ is non-empty for $G$. It follows that $m(G) \geq 3$ and $G$ is connected. By [5, 2.63], $O\left(C_{G}(t)\right)=1$ for any involution of $G$. This will be of particular importance in the final stages of the proof.

The following lemma is useful for a study of the 2-local subgroups.

LEMMA 1. Suppose $Q \triangleleft H, Q \in S_{2}(H), R \in S_{p}(H), \quad o(R)=r$, and that $H$ has no elements of order $2 r$.

If $P \in H\left(R, 2^{\prime}\right)$, then $[P, R] \subseteq O(H)$.

Proof. Suppose the lemma is false and let $H$ be a counterexample of minimum order.

First, $O(H)=1$. Otherwise setting $\bar{H}=H / O(H), \bar{H}, \bar{P}, \bar{R}$ satisfy the hypothesis of the lemma, whence $[P, R] \subseteq O(\bar{H})=\overline{O(H)}=1$ giving $[P, R] \subseteq O(H)$.

If $R$ centralizes all Sylow subgroups of $P$ the result is trivial. By the minimality of $O(H)$, we may assume $P$ is a $p$-group for some prime $p$ and $H=Q P R$. Put $R=\langle\phi\rangle$. If $P$ is abelian then $P=C_{P}(R) \times[P, R]$ and $\phi$ acts fixed-point-freely on $[P, R]$ and hence on $Q[P, R]$. It follows that $Q[P, R]=Q \times[P, R]$, whence $[P, R]=1$ contradicting our choice of $R$.

By [9, Theorem 5.3.8], $P$ is a non-abelian special p-group with $\phi$ acting irreducibly on $P / \Phi(P)$ and trivially on $\Phi(P)$. Further, $\Phi(Q)=1$ 
by Burnside's Lemma and the minimality of $O(H)$. Thus $Q=Q_{1} \oplus \ldots \oplus Q_{S}$ is elementary abelian with $Q_{i}$ irreducible under $P R$.

$$
\text { Put } P_{i}=C_{P R}\left(Q_{i}\right)=C_{p}\left(Q_{i}\right) \text {. As } P_{i} \text { is } \phi \text {-invariant, } P_{i}(P) / \Phi(P) \text { is }
$$
a $\phi$-invariant subspace of $P / \Phi(P) ; \phi$ acts irreducibly on $P / \Phi(P)$, whence $P_{i} \Phi(P)=\Phi(P)$ or $P$.

Assume $P_{i} \subseteq \Phi(P)$ for some $i$. Then $P R / P_{i}$ has a faithful irreducible representation on $Q_{i}$ and so $Z\left(P R / P_{i}\right)$ is cyclic. Since $Z\left(P R / P_{i}\right)=Z\left(P / P_{i}\right), P / P_{i}$ is extra-special of order $p^{2 r+1}$, say. By $[9$, Exercises, p. 37i-372], $Q$ has a minimum polynomial of $x^{r}-1$ on $Q_{i}$ or $p^{t}=r-1$. As $p$ and $r$ are odd, the latter is impossible, and so $\phi$ has an eigenvalue of $l$ in its representation on $Q_{i}$. Then $\phi$ fixes an involution, contrary to hypothesis.

We are left with $P_{i} \Phi(P)=P$ for each $i$, whence $P_{i}=P$ for each $i$. Thus $P$ centralizes $Q$ : a final contradiction which establishes the lemma.

Recently Glauberman ([7], [8]) has classified all simple groups which do not involve $\Sigma_{4}$, the symetric group on four letters. His work guarantees the existence of an element of order 3 in some 2-local subgroup in any counterexample to our theorem. To exploit this the following lemma is basic.

LEMMA 2. Let $H$ be a finite group with $H / O_{2}(H) \simeq \Sigma_{3}$. Suppose $H$ has no elements of order $6, P \in S_{2}(H)$, and $\phi$ is an element of order 3 in $H$. Then

(i) $\operatorname{cl}\left(\mathrm{O}_{2}(H)\right) \leq 2$,

(ii) if $R \leq \mathrm{O}_{2}(H)$ is abelian, $\left\langle R, R^{\phi}\right\rangle$ is also abelian,

(iii) if $O\left(\mathrm{O}_{2}(H)\right)>4$ then the class of $\mathrm{O}_{2}(H)$ is less than the class of any other subgroup of $P$ of index 2 , 
(iv) if $R \leq \mathrm{O}_{2}(H)$ is $\phi$-invariant and $s \in P \backslash \mathrm{O}_{2}(H)$, then $o\left(C_{R}(S)\right) \leq o(R)^{\frac{1}{2}}$.

Proof. Explicitly given in [20] as Proposition 2.2, although attributed, by Higman, to Burnside.

LEMMA 3. A non-soluble proper subgroup of $G$ has order prime to 3 iff it involves a Suzuki group.

Proof. By induction any non-soluble proper subgroup of $G$ with order prime to 3 must involve a Suzuki group.

For the converse, let $H$ be a proper subgroup of $G$ which involves a Suzuki group. Suppose $H$ has a chief series $H=H_{0} \triangleright H_{1} \triangleright \ldots \triangleright H_{n}=1$ with $H_{i} / H_{i+1}=\bar{H}_{i}=\bar{S}_{1} \times \bar{S}_{2} \times \ldots \times \bar{S}_{r}$, a direct product of isomorphic Suzuki groups. Let $R \in S_{3}\left(H_{i+1}\right)$. If $N(R)$ does not contain a fourgroup then a Sylow 2-subgroup of $N(R)$ is cyclic, generalized quaternion, or trivial. By the Frattini argument $N(R)$ involves a Suzuki group, whence $N(R)$ contains a subgroup $V R$ with $V$ a four-group. As $R=\prod_{x \in V^{\#}} C_{R}(x)$ and $G$ has no elements of ordex 6 , it follows that $R=1$, and it is sufficient to show that $\bar{H}=H / H_{i+1}$ contains no elements of order 3 .

Let the kernel of the action of $\bar{H}$ on the components of $\bar{H}_{i}$ be $\bar{K}$, and suppose that $\phi$ is an element of order 3 in $\bar{H}$. If $\phi \in \bar{G} \backslash \bar{K}$, then $\phi$ acts non-trivially on $\bar{S}_{1} \times \bar{S}_{2} \times \ldots \times \bar{S}_{p} \cdot$ For some $\bar{S}_{i}$ and some involution $t \in \bar{S}_{i}$ the subgroup $\bar{S}_{i} \times \bar{S}_{i}^{\phi} \times \bar{S}_{i}^{\phi^{2}}$ contains the involution $t \cdot t^{\phi} \cdot t^{\phi^{2}}$, which is centralized by $\phi$. If $\phi \in \bar{K}$, it normalizes each component, permuting the involutions in each one. The number of involutions in any component is clearly prime to 3 , and so $\phi$ centralizes at least one (in fact $\phi$ centralizes a Suzuki group). In either case we have an element of order 6 , contrary to hypothesis.

Suppose that $G$ has a non-soluble 2-local subgroup which contains an element of order 3 . In the following lemmas we investigate the structure 
of such a subgroup, eventually contradicting its existence. Thus choose $N$ to be a non-soluble 2-local subgroup with $3 / O(N)$. Further choose $N$ such that $o\left(O_{2}(N)\right)$ is maximal and put $Q=O_{2}(N)$.

LEMMA 4. $N$ has a section $M$ such that $M / O_{2}(M)$ is isomorphic to one of $L_{2}(q), L_{3}(q)$, and $U_{3}(q), O_{2}(M) \simeq O_{2}(N)$, and a sy low 2-subgroup of $M$ is isomorphic to a syzow 2-subgroup of $N$.

Proof. Let $K=\operatorname{sol}(N)$. If $R \in S_{3}(K)$, then $N(R)$ is non-soluble. Since $R$ is cyclic, $C(R)$ is non-soluble and $G$ contains an element of order 6 . Thus $R$ is trivial.

If $P \in S_{2}(K)$ then by the Frattini argument again, $N(P)$ is nonsoluble and contains a 3-element. By the maximality of $Q, P=Q$ and $K=O_{2,2^{\prime}}(N)$. Since we seek a section of $N$, we may assume $O(N)=I$.

Let $H / K$ be a minimal normal subgroup of $M / K$. By Lemma $3, H / K$ is isomorphic to one of $L_{2}(q), L_{3}(q)$, and $U_{3}(q)$. Put $\bar{H}=H / O_{2}(H)$. Let $\phi$ be an element of order 3 in $H, L$ a $\phi$-invariant complement of $O_{2}(H)$ in $O_{2,2^{\prime}}(H)$. Applying Lemma 1 to $Q L(\phi),[L, \phi] \subseteq O(H)=1$, whence $C_{\bar{H}}(\bar{L}) \cdot \bar{L}>\bar{L}$ forcing $H=C_{\bar{H}}(\bar{L}) \cdot \bar{L}$. As each of $L_{2}(q), L_{3}(q)$, and $U_{3}(q)$ has a multiplier which is, at worst, a $\{2,3\}$-group [2], $\bar{H}=\bar{M} \times \bar{L}$, where $\bar{M} \simeq L_{2}(q), L_{3}(q)$, or $U_{3}(q)$, and $\bar{L}=O(\bar{H})$. As $\bar{M}$ has an element of order 3 , so $C \bar{N}(\bar{M})$ has odd order and thus equals $\bar{L}$. It follows that $\bar{H} / \bar{L} \leq \operatorname{aut}(\bar{M})$. All outer automorphisms of $\bar{M}$ of even order fix elements of order 3 in $\bar{M}$. Thus $[\bar{N}: \bar{M}]$ is odd and the required properties of $M$ follow easily.

LEMMA 5. $M / O_{2}(M) ‡ L_{3}(q)$ or $U_{3}(q)$.

Proof. Put $\bar{M}=M / O_{2}(M)$. Let $\varepsilon=1$ (respectively -1 ) if $\bar{M} \simeq L_{3}(q) \quad\left(\right.$ respectively $\left.U_{3}(q)\right)$ and $\nu=(3, q-\varepsilon) \cdot \bar{M}$ contains a cyclic Hall subgroup $\vec{D}, o(\bar{D})=\left(q^{2}+\varepsilon q+1\right) / v$ with $\left[N_{M}(\bar{D}): \because \bar{D}\right]=3(\bar{D}$ is a singercycle $[17]$, Satz 7.3$)$, and $(o(\bar{D}), 3)=I$. If $\phi$ is an element of order 3 in $N_{M}(\bar{D})$ then $\bar{D}=C \bar{D}(\phi) \times[\bar{D}, \phi]$ and $\phi$ acts fixed-point-freely on 
$[\bar{D}, \phi]$ and so on $[\bar{D}, \phi] O_{2}(M)$. It follows that $[D, \phi] O_{2}(M)$ is nilpotent and $C \bar{M}\left(O_{2}(M)\right)$ is non-trivial. Thus $C{ }_{M}\left(O_{2}(M)\right)=\bar{M}$ and we have an element of order $6:$ a contradiction.

LEMMA $6 . M / O_{2}(M) \neq L_{2}(q), q$ odd.

Proof . By [20], Proposition 3.1.

LEMMA 7. $M / O_{2}(M) \neq L_{2}\left(2^{n}\right)$.

Proof. By [20], Proposition 4.l, if $P \in S_{2}(M)$, then $c l(P)=2$. If $o\left(o_{2}(M)\right)>2^{n}$ or $n \geq 3$ then $O_{2}(M)$ char $P$, ([20], Proposition 4.1, 5.4). It follows that $P \in S_{2}(G), G$ has Sylow 2-subgroups of class 2 . The classification of all such groups has been completed $([5,6])$ and no counter-example exists in their list.

Thus we may assume $M / O_{2}(M) \simeq L_{2}(4) \simeq A_{5}, O_{2}(M) \simeq E_{16} \cdot$ Let $P \in S_{2}(M), \phi$ a 3 -element in $N(P)$, and $t$ an involution in $P \backslash O_{2}(M)$ (otherwise $O_{2}(M)=\Omega_{1}(P)$ char $P$ and we are finished as above). Now $t$ and $t^{\phi}$ commute, so $P$ is the split extension of $E_{16}$ by $A_{5}$. Only two such groups exist [19], and one contains elements of order 6 , whence $M$ is completely determined. A Sylow 2-subgroup of $M$ and hence of $N$ is isomorphic to that of $\operatorname{PSL}(3,4)$ : we need only note that it contains precisely two elementary abelian normal subgroups of order 16 , one of which is $O_{2}(M)=Q$, which contain all the involutions of the Sylow 2-subgroup.

Let $P \in S_{2}(N), \quad R \in S_{2}(N(P))$. If $P=R$ then $P \in S_{2}(G)$, $c l(P)=2$, and $G$ is known. As $R$ acts on the two elementary abelian subgroups of $P$ of order 16 , with kernel of the action in $N(Q)$, we have $[R: P]=2$. As $3 \mid O(N(P))$ we can choose $R<X \leq N(P)$ with $X / P \simeq \Sigma_{3}$ and the extension splits by a Sylow argument. By Lemma 2 ( $i i i$ ), $P$ char $R$, whence $R \in S_{2}(G)$. If $t$ is an involution in $R \backslash P, t$ interchanges $Q$ and $Q^{t}$. Now $Q \cap Q^{t}=Z(P)=\Phi(P)$ admits a 3 -element 
in its normalizer, and by Lemma $2(i v),\left|C_{Z(P)}(t)\right| \leq|Z(P)|^{\frac{3}{2}}=2$. Thus $t$ interchanges two involutions in $Z(P)$. Checking the outer automorphism group of $P$, we see that $R$ is now completely determined. The arguments of Gorenstein and Harada [10, pp. 354-355] quickly produce an element of order 6 and finish the proof.

COROLLARY 7. If a 2-local subgroup has order divisible by 3 , it is soluble.

LEMMA 8. Let $S \in S_{2}(G)$. Then $S$ contains a characteristic subgroup $S_{0}$ of index 2 with $c 1\left(S_{0}\right) \leq 2$. Moreover, $3 / 0\left(N\left(S_{0}\right)\right)$.

Proof. By Glauberman, some 2-group of $G$ has an element of order 3 in its normalizer. Let $Q$ be such a 2-group with maximal order and put $N=N(Q)$. By the Corollary 7, $N$ is soluble and by the maximality of $O(Q)$ and the Frattini argument $Q=O_{2}(N)$. Let $R \in S_{3}\left(O_{2,2^{\prime}}(N)\right)$,

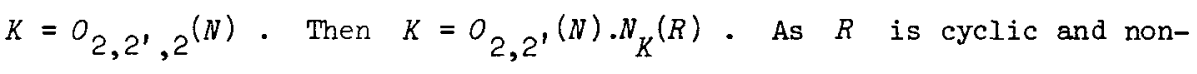
trivial, $N_{K}(R)$ has a Sylow 2-subgroup of order 2 and hence a subgroup of index $2^{\prime}$. It is immediate that $0_{2,2^{\prime}, 2,2^{\prime}}(N)=0_{2,2^{\prime}, 2^{(N)}=N}$. Moreover there exists $H \leq N$ with $H / Q \simeq \Sigma_{3}$. If $O(Q)=4$, then $Q$ is elementary abelian and self-centralizing in any Sylow 2-subgroup of $G$ in which it appears. The sylow 2-subgroups of $G$ are then dihedral or semidihedral [21] and $G$ is known [1] and $[13,14,15]$. No counterexample to our theorem lies in their list, whence $O(Q)>4$. If $P \in S_{2}(N)$, then $Q \operatorname{char} P$ by Lemma 2 (iii). Thus $P \in S_{2}(G)$, and the lemma follows from Lemme 2 (ii), (iii).

COROLLARY 8. There are no perfect central extensions of $S z(8)$ involved in $G$.

Proof. [2] gives generators and relations for the perfect central extensions of $S z(8)$. In all cases the Sylow 2-subgroup has class 3 and no subgroup of index 2 has class 2 .

REMARK. For the remainger of the proof, if $S \in S_{2}(G)$ then $S_{0}$ will denote the subgroup given in Lemma 8 . Moreover, when no confusion is possible, if $X \leq S$ then $X_{0}$ will denote $X \cap S_{0}$. 
LEMMA 9. $N(Q) / C(Q)$ is soluble for any 2-group $Q$ of $G$.

Proof. Suppose that $Q$ is a 2-group of $G$ maximal subject to $N(Q) / C(Q)$ being non-soluble. Put $T=\operatorname{sol}(N \bmod C(Q))$, where $N=N(Q)$ and let $K=\operatorname{soc}(N \bmod T)$. Let $Q_{1} \in S_{2}(T)$. Then $N / T \simeq N\left(Q_{1}\right) / N_{T}\left(Q_{1}\right)$ is non-soluble, $C\left(Q_{1}\right) \subseteq N_{T}\left(Q_{1}\right)$ and by the maximality of $Q$, $Q=O_{2}(N) \in S_{2}(T)$, and $K / T$ is a Suzuki group. It follows that $C(Q)$ is soluble and $N / K$ has odd order.

Suppose that $Q \subset P \subseteq S$, with $P \in S_{2}(N), S \in S_{2}(G)$. Now $Q_{0} \subset P_{0}$ and $P_{0}^{\prime} \subseteq Z\left(P_{0}\right)$, whence $Q_{0} \subseteq C\left(P_{0}^{\prime}\right)$ and $\left[Q: C_{Q}\left(P_{0}^{\prime}\right)\right] \leq 2$. Choose another sylow 2-subgroup $P^{*}$ of $N$ such that $\left(P, P^{*}\right\rangle$ involves a Suzuki group. Suppose that $P^{*} \subseteq S^{*} \in S_{2}(G)$, and set $P_{0}^{*}=S_{0}^{*} \cap P^{*}$. As above $\left[Q: C_{Q}\left(P_{0}^{* \prime}\right)\right] \leq 2$ and $\left\langle P_{0}^{\prime}, P_{0}^{* \prime}\right\rangle$ still involves a Suzuki group and centralizes $C_{Q}\left(P_{0}^{\prime}\right) \cap C_{Q}\left(P_{0}^{* \prime}\right)$. But this latter group has index at most 4 in $Q$, whence $C_{G}(Q)$ involves a Suzuki group: a contradiction.

Gorenstein and Lyons have recently classified all simple groups with soluble 2-local subgroups [12]. Consequently $G$ contains some nonsoluble 2-local subgroups and, by Lemma 9, an involution with a non-soluble centralizer.

Let $A$ be an elementary abelian 2-group of $G$ maximal subject to (*) there exists $a \in A$ such that $C_{G}(\dot{a})$ involves a suzuki group.

For the remainder of the proof we fix the following notation: $H=C_{G}(\alpha), T \in S_{2}(H), S \in S_{2}(G), A \leq T \leq S$, and $\phi$ is an element of order 3 in $N\left(S_{0}\right)$.

LEMMA 10. We can choose $a, A$ satisfying (*) and $A$ maximal and have $A \leq T_{0}$ and $A$-invariant. In particular $3 \mid O(N(A))$.

Proof. If $A \leq T_{0}$ then $\left\langle A, A^{\phi}\right\rangle$ is abelian by Lemma 2 (ii), and hence elementary abelian. By the maximality of $O(A), A=\left\langle A, A^{\phi}\right\rangle$ is $\phi$-invariant. Thus we may assume $A>A_{0}$. 
Suppose we can choose $a \in A_{0}$. Then $\left\langle A_{0}, A_{0}^{\phi}\right\rangle$ is elementary abelian and the maximality of $r(A)$ gives $r\left(A_{0}\right) \leq r\left(\begin{array}{ll}A_{0} & A_{0}^{\phi}\end{array}\right)+1 \cdot A_{0} \cap A_{0}^{\phi}$ is $\phi$-invariant and centralized by any $t \in A \backslash A_{0}$. By Lemma 2 (iv), $A_{0} \cap A_{0}^{\phi}=1, \quad o\left(A_{0}\right)=2, \quad o(A)=4$. As $o\left[\left\langle A_{0}, A_{0}^{\phi}\right\rangle\right)=4$, we can change our choice of $a, A$ to satisfy the lemma.

Arguing by contradiction we may assume that there is no choice of $a$ such that $a \in A_{0}$. Now $\langle a\rangle \triangleleft S, S_{0} \triangleleft s$, whence $S=\langle a\rangle \times S_{0}$ and $H=\langle a\rangle \times H_{0}$, say. $L(H \delta$ is a central product of Suzuki groups. By [2], the only Suzuki group which admits a perfect central extension is $S z(8)$, and by Corollary 8 this does not appear. Thus $L\left(H_{0}\right)$ is a direct product. Moreover $A=B \times\langle a\rangle$ where $S_{0} \geq B \in M\left(H_{0}\right)$. Let $t \in S_{2} \backslash L(H)$ and suppose $t$ interchanges two components $K_{1}$ and $K_{2}$ of $L\left(H_{0}\right)$. Choosing $x, y \in R \in S_{2}\left(K_{1}\right)$ with $[x, y] \neq 1$, we have $[x, y, t] \neq 1$ contrary to $\operatorname{cl}\left(S_{0}\right)=2$. Thus $S_{0}$ normalizes each component of $L\left(H_{0}\right)$. Moreover all outer automorphisms of Suzuki groups have odd order, whence $S_{0}=U \times V$, with $U \in S_{2}\left(C_{H_{0}}\left(L\left(H_{0}\right)\right)\right)$ and $V \in S_{2}\left(L\left(H_{0}\right)\right)$. Thus $B$ is a direct product of a member of $E(U)$ and a direct product of centres of sylow 2-subgroups of suzuki groups. If $U$ is non-trivial we could choose $a \in U<A$ to satisfy the lemma and similarly if $L\left(H_{0}\right)$ has more than one component. Since $O(H)=1$ by an earlier remark, $C_{G}(a)=H=\langle a\rangle \times K$, where $K \leq \operatorname{aut}(S z(q))$. The result of Dempwolff [3] completes the proof.

LEMMA 11. (i) $L(H) C_{H}(L(H))=L(H) \times C_{H}(L(H))$.

(ii) $L(H)$ is a direct product of Suzuki groups,

$$
K_{1} \times K_{2} \times \ldots \times K_{r} .
$$

(iii) Each $K_{i}$ is A-invariant.

(iv) $A \cap K_{i} \in M\left(K_{i}\right)$, that is $A \cap K_{i}=Z\left(R_{i}\right), R_{i} \in S_{2}\left(K_{i}\right)$. 
Proof. The arguments of Lemma 10 give (i) and (ii).

Choosing $t \in A$ and knowing $t \in T_{0}$ we can argue as in Lemma 10 with $K_{i}$ and $K_{i}^{t}$ to prove $(i i i)$.

Now $A \in M(H)$ whence $A \in M\left(A K_{i}\right)$. From $A K_{i}=C_{A}\left(K_{i}\right) \times K_{i}$ it follows immediately that $A \cap K_{i} \in M\left(K_{i}\right)$ and (iv) is true.

Completion of the proof. By Lemma 10, $\phi \in N_{G}(A) \backslash C_{G}(A)$ whence $\phi \in \mathbb{N}_{G}(S)$ for some $S \in S_{2}\left(C_{G}(A)\right)$.

Let $K$ be a component of $H$ with $R \in S_{2}(K), R>Z(R)=A \cap K$. Let $t \in C_{G}(A)$. Then $K \cap K^{t} \geq A \cap K$, whence $K=K^{t}, R=R^{t}$, and $R \leq S$. Moreover $S \subseteq C_{G}(A) \leq N_{H}(K)$ and, as $\mid$ aut $K: K \mid$ is odd, $S \subseteq C_{H}(K) . K=C_{H}(K) \times K$. If we write $S=S_{1} \times S_{2} \times \ldots \times S_{q}$ with $S_{i}$ indecomposable, then we may assume by the above argument that $R=S_{1}$. By the Krull-Schmidt Theorem, $\phi$ permutes the derived groups $S_{i}^{\prime}$. If $\phi$ fixes $S_{1}^{\prime}$, it permutes the $2^{2 n+1}-1$ involutions in the group and so fixes at least one. On the other hand, choosing $t \in S_{1}^{\prime}$, $S_{1}^{\prime} \times\left(S_{1}^{\prime}\right)^{\phi} \times\left(S_{1}^{\prime}\right)^{\phi^{2}}$ contains the involution $t \cdot t^{\phi} \cdot t^{\phi^{2}}$ fixed by $\phi$. In either case we have an element of order 6 contrary to assumption.

\section{References}

[1] J.L. Alperin, Richard Brauer and Daniel Gorenstein, "Finite groups with quasidihedral and wreathed Sylow 2-subgroups", Trans. Amer. Math. Soc. 151 (1970), 1-26.

[2] J.L. Alperin and Daniel Gorenstein, "The multiplicators of certain simple groups", Proc. Amer. Math. Soc. 17 (1966), 515-519. 
[3] Ulrich Dempwolff, "A characterization of the Rudvalis simple group of order $2^{14} \cdot 3^{3} \cdot 5^{3} \cdot 7 \cdot 13.29$ by the centralizers of noncentral involutions", J. Algebra 32 (1974), 53-88.

[4] L.R. Fletcher, B. Stellmacher, W.B. Stewart, "Endliche Gruppen, die kein Element der Ordung 6 enthalten", (unpublished).

[5] Robert Gilman and Daniel Gorenstein, "Finite groups with Sylow 2-subgroups of class two. I", Trans. Amer. Math. Soc. 207 (1975), 1-101.

[6] Robert Gilman and Daniel Gorenstein, "Finite groups with Sylow 2-subgroups of class two. II", Trans. Amer. Math. Soc. 207 (1975), 103-126.

[7] George Glauberman, "Factorizations for 2-constrained groups", preprint.

[8] George Glauberman, "A characterization of the Goldschmidt groups", (unpublished).

[9] Daniel Gorenstein, Finite groups (Harper and Row, New York, Evanston, London, 1968).

[10] Daniel Gorenstein and Koichiro Harada, "A characterization of Janko's two new simple groups", J. Fac. Sci. Univ. Tokyo Sect. I 16 (1969), 331-406 (1970).

[11] Daniel Gorenstein and Koichiro Harada, Finite groups whose 2-subgroups are generated by at most 4 elements (Memoirs Amer. Math. Soc., 147. Amer. Math. Soc., Providence, Rhode Island, 1974).

[12] Daniel Gorenstein and Richard Lyons, "Nonsolvable finite groups with solvable 2-local subgroups", J. Algebra 38 (1976), 453-522.

[13] Daniel Gorenstein and John H. Walter, "The characterization of finite groups with dihedral Sylow 2-subgroups. I", J. Algebra 2 (1965), 85-151.

[14] Daniel Gorenstein and John H. Walter, "The characterization of finite groups with dihedral Sylow 2-subgroups - II", J. Algebra 2 (1965), 218-270. 
[15] Daniel Gorenstein and John $H$. Walter, "The characterization of finite groups with dihedral Sylow 2-subgroups. III", J. AZgebra 2 $(1965), 354-393$.

[16] Daniel Gorenstein and John H. Walter, "Centralizers of involutions in balanced groups", J. AZgebra 20 (1972), 284-319.

[17] B. Huppert, Endliche Gmppen I (Die Grundlehren der mathematischen Wissenschaften, 134 . Springer-Verlag, Berlin, Heidelberg, New York, 1967).

[18] R. Patrick Martineau, "On simple groups of order prime to 3 ", Proc London Math. Soc. (3) 25 (1972), 213-252.

[19] Charles C. Sims, "Computational methods in the study of permutation groups", Computational problems in abstract algebra, 169-183 (Proc. Conf. Oxford, 1967. Pergamon Press, Oxford, London, Edinburgh, New York, Toronto, Sydney, Paris, Braunschweig, 1970).

[20] W.B. Stewart, "Groups having strongly self-centralizing 3-centralizers", Proc. London Math. Soc. (3) 26 (1973), 653-680.

[21] Michio Suzuki, "A characterization of simple groups $\operatorname{LF}(2, p) ", J$. Fac. Sci. Univ. Tokyo Sect. I 6 (1951), 259-293.

[22] John H. Walter, "The characterization of finite groups with abelian Sylow 2-subgroups", Amm. of Math. (2) 89 (1969), 405-514.

Department of Pure Mathematics, University of Sydney,

Sydney,

New South Wales. 